\title{
Review of: "GABAergic basal forebrain projections to the periaqueductal gray promote food consumption, reward and predation"
}

\author{
Newton Canteras ${ }^{1}$ \\ 1 Universidade de São Paulo
}

Potential competing interests: The author(s) declared that no potential competing interests exist.

This study brings important insights with respect to understanding the neural basis of an important common eating disorder characterized by recurrent periods of eating large amounts of food in the absence of physical hunger, called the binge eating disorder. A plausible cause for this eating disorder is dysregulation of the eating mechanisms that lead to food consumption even in the absence of homeostatic drive. In this work, Roman-Ortiz and colleagues explored a putative path consisting of the basal forebrain and the periaqueductal gray that are likely to be involved in eating behaviors dissociated from the homeostatic drive for food consumption.

The authors examined the activation of basal forebrain (BF) GABAergic neurons in consummatory behaviors. Accordingly, using GAD2-Cre mice and Cre-dependent adeno-associated virus (AAV) expressing channelrhodopsin (ChR2), the authors were able to stimulate GABAergic neurons in the BF, which encompassed the ventral pallidum, substantia innominata and horizontal diagonal band. The authors demonstrated that activation of BF GABAergic neurons promotes consummatory behavior that is directed at both food and non-food items, and therefore is independent of nutritional characteristics associated with consumption in addition to inducing fictive eating. Stimulation of these cells also showed clear reward-like motivational effects that induced place preference and disrupted defensive threat responses. The authors also explored how BF GABAergic neurons influence on predatory behavior. In this regard, the authors provide a very ingenious comparison among live and artificial prey in addition to a remote-controlled toy car. In all situations, activation of BF GABAergic neurons triggered an immediate attack that depended on prey/toy movement to provide feedback essential for the predatory effects of BF GABAergic activation. Finally, after testing social interaction, the authors found that BF GABAergic activation did not trigger aggression toward novel conspecifcs of either sex, suggesting a specific form of aggression that is integral to feeding.

As the authors pointed out, the BF is a very heterogenous region, and it would be important to determine which element(s) in the BF would be more likely to influence consummatory drive responses examined in this study. This information would be particularly valuable for tracing potential sources of inputs involved 
in the controlling this form of eating behavior and to have a more complete overview of the neural system underlying these responses. Moreover, it is important to consider that photostimulation synchronizes a large population of cells and is likely to results in an artificial response, which is unlikely to occur under natural circumstances. In this way, it would be important to determine to what extent the BF GABAergic neurons are activated under situations of increased consummatory drive dissociated from the homeostatic drive, such as during predatory hunting in well-fed animals, and how the inactivation of this neuronal population could interfere with these responses. In the same vein, it would be interesting to evaluate possible dysfunction of BF GABAergic neurons in patients suffering from binge eating disorder.

In addition, the authors showed that selective stimulation of BF > Periaqueductal Gray (PAG) GABAergic projections recapitulated the same consummatory drive responses previously obtained with BF GABAergic neuron activation, including food consumption, fictive eating, and predatory hunting in addition to strong place preference. It is still quite speculative how PAG mediates these responses. As pointed out, BF GABA neurons project particularly to the lateral and ventrolateral PAG divisions. Previous studies have shown that direct inactivation of the ventrolateral PAG activity leads to food intake and prey hunting. In fact, the stimulation of GABAergic projections from either the lateral hypothalamus [1] or the central amygdala [2] was shown to drive a strong predatory attack. Likewise, it has also been shown that suppression of GABAergic cell activity in the anterior ventrolateral periaqueductal gray is sufficient to promptly induce feeding behavior in well-fed mice [3]. Conversely, recent studies suggest that GABAergic neurons in the lateral hypothalamus facilitate predation in part by suppressing defensive responses to prey that are encoded in the periaqueductal gray [4]. At this point, it is not clear how inhibition of the ventrolateral PAG could induce feeding and predatory hunting. Functional studies have revealed that predatory hunting is associated with activation of glutamatergic cells in the lateral PAG [5, 6]. To reconcile the findings of inhibitory drive activation in the ventrolateral PAG and glutamatergic cell activation in the lateral PAG during predatory hunting, it is plausible to suggest the existence of disinhibitory mechanisms in the PAG that should be considered for inducing predatory hunting and perhaps feeding responses also.

The work of Roman-Ortiz and colleagues corroborates the findings of inhibitory drive to the PAG that induces feeding and predatory hunting and provides an important piece of information to expand our knowledge on the neural control of such responses in addition to opening insightful perspectives to decipher mechanisms underlying the binge eating disorder.

1. Li, Y. et al. Hypothalamic circuits for predation and evasion. Neuron 97(4), 911-924.e5 (2018)

2. Han, W. et al. Integrated control of predatory hunting by the central nucleus of the amygdala. Cell 168(1), 311-324.e18 (2017)

3. Hao, S. et al. The lateral hypothalamic and BNST GABAergic projections to the anterior ventrolateral periaqueductal gray regulate feeding. Cell Rep. 28(3), 616-624.e5 (2019). 
4. Rossier, D. et al. A neural circuit for competing approach and defense underlying prey capture. Proc. Natl. Acad. Sci. 118(15), e2013411118 (2021).

5. Predatory hunting and exposure to a live predator induce opposite patterns of Fos immunoreactivity in the PAG.

6. Comoli, E. et al. Predatory hunting and exposure to a live predator induce opposite patterns of Fos immunoreactivity in the PAG. Behav Brain Res. 138(1):17-28 (2003)

7. Marín-Blasco, I. et al. The lateral periaqueductal gray and its role in controlling predatory hunting and social defense. bioRxiv (2020) 